\title{
Fluorescence studies of a silane gel to be applied as a carrier in optical sensors
}

\author{
Ewa Miller, ${ }^{\dagger}$ Stanisław Wysocki, and Donata Jóźwik \\ Institute of General Food Chemistry, Technical University of Łódź, \\ ul. Stefanowskiego 4/10, 90-924 Łódź, Poland
}

\begin{abstract}
Results of fluorescence studies on the degree of pyrene and aminopyrene elution from a silane gel by water and ethanol are discussed in the paper. The studies were carried out for gels obtained on glass plates that differed in composition. Silane monomers: TEOS-tetraethylorthosilane, APTES-3-aminopropyltriethoxysilane, GPTMS-3-glycidoxypropylmethoxysilane, PDMS-polydimethylsilane, TMAOHtetraethylammonium hydroxide, water and ethanol were used. A fluorophore was trapped in the gel either physically or using covalent bonds with the amino group of a carrier and glutaraldehyde. Emission spectra of the probe steady-state fluorescence in the gel were recorded on subsequent days of storage of plates with the gel in fresh solvent. The kinetics of aminopyrene decay in the sol and silane gel was also recorded. On the basis of results obtained, the degree of elution of fluorophore from the gel was determined in view of the application of the silane gel as a carrier in the optical sensor and the nature of the probe environment.
\end{abstract}

\section{INTRODUCTION}

High requirements of modern biomedicine and biotechnology have become an inspiration for a new generation of analytical optical methods that use optical sensors. A general requirement for most optical sensors is to immobilize an indicator on fibers. The color or luminescence spectrum of the indicator changes due to interactions with a substance and is a measure of tested properties. Recently, a number of studies have been published on optical indicators, immobilized in the silane gel obtained by the sol-gel method [1-3]. The gel is frequently used as a carrier of organic fluorophore because of a simple method of its formation and low polycondensation temperature.

An important prerequisite for applying the carrier with immobilized fluorophore in the optical sensor construction is stability of the system during the electrode operation which is connected with a permanent fluorophore immobilization. It is important that the indicator located in the gel is not eluted. Therefore, in the studies on optode construction, a variety of techniques were used to locate fluorophore in the gel matrix [4]. In this paper results of studies on pyrene and aminopyrene elution from the silane gel with three different compositions are discussed. Pyrene was trapped in the silane gel physically, while aminopyrene was trapped by means of a chemical bond, using glutaraldehyde as a "spacer". The fluorophore elution from the gel in solvents with different polarity was investigated using a test that covered a long-term storage of the plates with gel in proper liquids. The effect of $\mathrm{pH}$ of water solutions on this fluorophore elution from silane matrices was also studied.

†E-mail: emiller@snack.p.lodz.pl

\section{EXPERIMENTAL}

Tetraethylorthosilane (TEOS) (Sigma), 3-aminopropyltriethoxysilane (APTES) (Aldrich), 3-glycidoxypropylmethoxysilane (GPTMS) (Aldrich), polydimethylsilane (PDMS) (Aldrich), tetraethylammonium hydroxide (TMAOH) (25\% wt water solution, Aldrich), pyrene, 1-aminopyrene (Aldrich), anhydrous ethyl alcohol (spectrally pure, P.O.Ch., Gliwice, Poland), hexane (Sigma-Aldrich), sodium hydroxide (analytically pure, Chempur), glutaraldehyde (Sigma).

Glass plates $(12 \mathrm{~mm} \times 45 \mathrm{~mm} \times 1.25 \mathrm{~mm})$ from special glass (Hellma).

Three types of sols were prepared:

Sol I: tetraethylorthosilane (TEOS), ethanol and water in the molar ratio 1:6:6. Pyrene was introduced to the sol in the solution of ethanol at the concentration $8 \cdot 10^{-5} \mathrm{M}$. After 2 hours of mixing, a sol was obtained at $\mathrm{pH} 4.3$, which gelated during 4-5 days.

Sol II: TEOS, APTES and PDMS in molar ratio 11 : $10: 1$, which contained pyrene at the concentration 8 . $10^{-5} \mathrm{M}$ or aminopyrene at the concentration $5 \cdot 10^{-5} \mathrm{M}$. The sol was prepared according to the procedure described elsewhere [1]. The sol stored at the temperature $5{ }^{\circ} \mathrm{C}$ did not gelate for dozens of days.

Sol III: TEOS, APTES, GPTMS and PDMS in molar ratio $11: 5: 5: 1$. The sol was also prepared according to the previous procedure [1]. The sol was characterized by high viscosity; it gelated in several hours. When aminopyrene was immobilized in the gel, glutaraldehyde was added to silane components to form covalent amide bonds between the amino groups of APTES and aminopyrene, and the aldehyde groups of glutaraldehyde.

Prior to sol deposition, the glass plates were soaked for 3 hours in $40 \%$ water solution of $\mathrm{NaOH}$, washed in 
Table 1. Physical properties of applied silane carriers.

\begin{tabular}{|c|c|c|c|c|c|c|}
\hline \multicolumn{2}{|c|}{ Composition } & \multirow{2}{*}{$\frac{\mathrm{I}}{\mathrm{P}}$} & \multicolumn{2}{|c|}{ II } & \multicolumn{2}{|c|}{ III } \\
\hline \multicolumn{2}{|c|}{ Name of probe } & & $\mathrm{P}$ & $\mathrm{AP}$ & $\mathrm{P}$ & $\mathrm{AP}$ \\
\hline \multicolumn{2}{|c|}{ Adherence [\%] } & 100 & 100 & 70 & 100 & 35 \\
\hline \multicolumn{2}{|c|}{ Transparency [\%] } & 100 & 100 & 35 & 100 & 35 \\
\hline \multirow{3}{*}{$\begin{array}{l}\text { Methods of } \\
\text { deposition [\%] }\end{array}$} & A & - & - & 35 & - & 35 \\
\hline & B & - & - & 100 & - & 100 \\
\hline & $\mathrm{C}$ & 100 & 100 & 70 & 100 & 70 \\
\hline
\end{tabular}

I TEOS, ethanol, water.

II TEOS, APTES, PDMS, TMAOH, ethanol.

III TEOS, PDMS, APTES, GPTMS, TMAOH, ethanol.

A: Scotch tape.

B: thin.

C: needle.

$100 \%$ transparent film, smooth, without cracks, very good adhesion to the surface of glass.

70\% transparent film, smooth, with fine cracks on the film edges, good adhesion to the surface of glass.

$35 \%$ transparent film, smooth, with cracks, weak adhesion to the surface of glass.

deionized water and dried at the temperature $150^{\circ} \mathrm{C}$ for around 2 hours. Onto so prepared plates sol was deposited by the three techniques and left for 3 days at the temperature around $20^{\circ} \mathrm{C}$.

Sol deposition techniques: A- "Scotch tape"-the sol was placed in a window formed as a result of sticking to the glass surface three layers of the Scotch tape, whose thickness was $130 \mu \mathrm{m}, \mathrm{B}$ the so-called "thin"the sol layer was deposited with a needle and distributed using a metal roller, C- "needle"-the sol was deposited and distributed using a preparative needle. The plate with gel on it was soaked in $3 \mathrm{ml}$ solvent, taken out every day to make measurement, and immersed again in a fresh liquid.

A Fluoromax-2 spectrofluorimeter (Jobin Yvon) was used to record the fluorescence emission spectra of the probe placed in different gels located on the plates and fluorescence emission spectra for solutions after soaking the plates with gels. The amount of eluted probe was calculated from the analytical curves of pyrene and aminopyrene in water and ethanol. Aminopyrene decay kinetics was determined using a time-resolved measuring system (Edinburgh Analytical Instruments Co.).

\section{RESULTS AND DISCUSSION}

Physical properties of the gels on plates are evaluated in Table 1.

Addition of APTES to initial sols II and III guaranteed the presence of amino groups in the gel net and an increase of the hydrophilic nature of the formed carrier surface. GPTMS and PDMS induced a growth of elasticity and porosity of the formed gel and an increase of the number of hydroxyl groups [1]. Plates with gels I, II and III, containing pyrene as a fluorophore, were transparent, the gel layer was characterized by very good adherence to the glass surface during long tests in ethanol and water. The best results were obtained for gel III. After a month of soaking the plates in water and ethanol, the gels remained transparent and well adhered to the glass surface.

After subsequent days of soaking gel II with pyrene on the plate in a fresh portion of water, a decrease of fluorescence intensity of the probe was recorded (Figure 1A). The nature of the spectrum indicated a monomeric form of the probe in the carrier. Storage of the plate with gel II in ethanol led to an increase of pyrene fluorescence, after initial drop, a rise in intensity and to the appearance of a band with a maximum at wavelength $\lambda=420 \mathrm{~nm}$ (Figure 1B). Probably, next portions of ethanol induced diffusion of the pyrene molecules from deeper layers of the gel to the surface, the result of which was a significant increase of local pyrene concentration and formation of dimers [5-8]. On the basis of the tendency towards a decrease of emission intensity of the probe fluorescence in the gel stored in water and ethanol, it can be concluded that pyrene is gradually eluted from the carrier pores. Despite this, after 57 days of soaking, in a fresh portion of the solvent every day, the pyrene was present in the gel and had 6 to 7 times lower fluorescence intensity as compared to initial values.

Aminopyrene (AP) was chosen as a fluorescence probe to investigate sol-gel transition because of the presence of amino group in the molecule. The amino group can participate in covalent bonding with APTES monomer which is contained in the sol. Additionally, this probe-if adsorbed on the silane carrier surfacecan be used to determine the nature of - $\mathrm{OH}$ group on this surface (vicinal or geminal) [9-11]. It can occur in two forms: protonated and neutral, depending on the polarity of a microenvironment, according to the formula:

$$
\mathrm{APH}^{+} \leftrightarrows \mathrm{AP}+\mathrm{H}^{+}
$$

These forms of aminopyrene differ in the shape of fluorescence emission spectrum [9]. On the basis of previous studies, it was found that by analogy to 

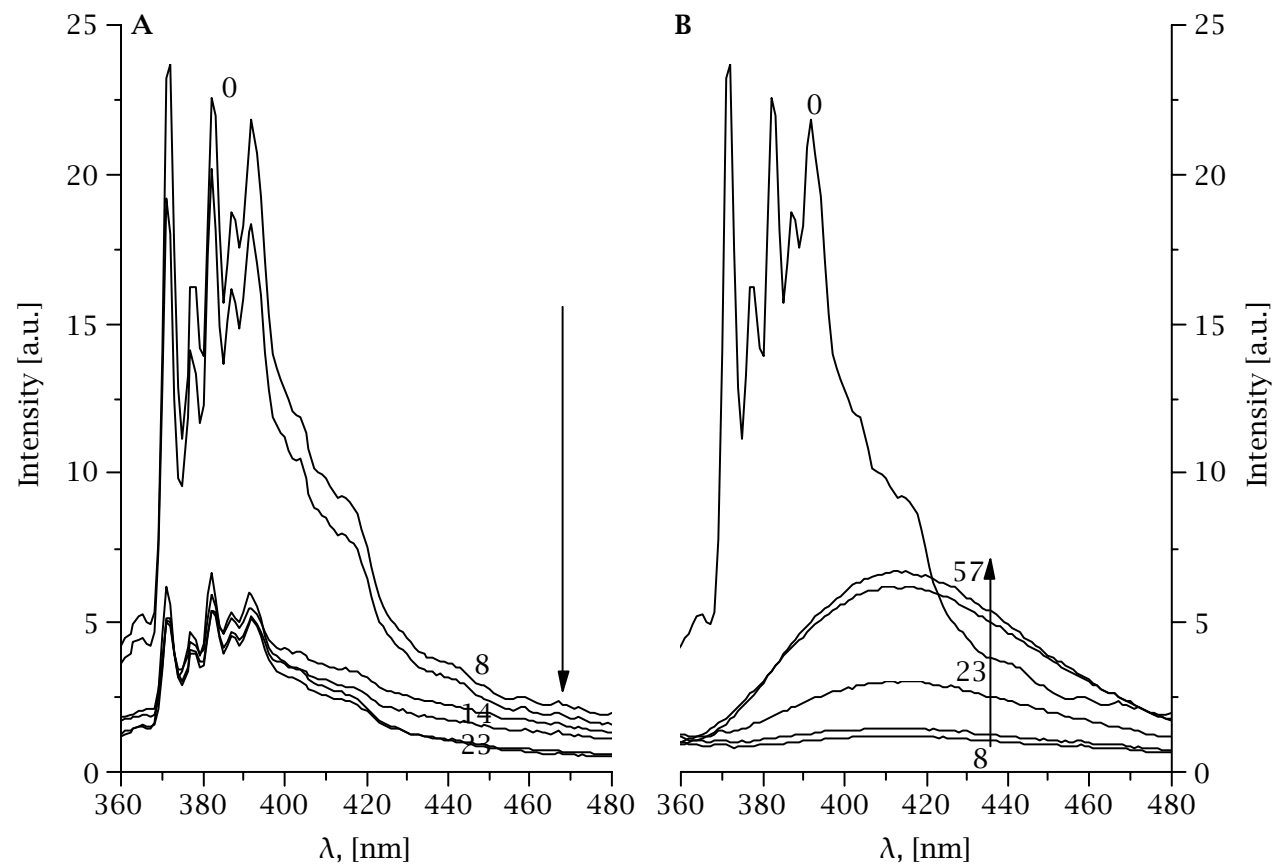

Figure 1. Fluorescence emission spectrum of pyrene in gel II on the plate stored in (A) water: 0-before soaking, 8, 12, 14, 19, 23-subsequent days of soaking, every day in fresh water; in (B) ethanol: 0-before soaking, 7, 8, 10, 23, 30, 57-subsequent days of soaking, every day in fresh ethanol.

Table 2. Aminopyrene lifetime for different forms of silane carrier, $\mathrm{pH}=4.2, c_{\mathrm{AP}}=2.1 \cdot 10^{-5} \mathrm{M}, \lambda_{\mathrm{em}}=430 \mathrm{~nm}$.

\begin{tabular}{lcccccc}
\hline Form & Amplitude & $\tau_{1}[\mathrm{~ns}]$ & $\%$ & Amplitude & $\tau_{2}[\mathrm{~ns}]$ & $\%$ \\
\hline Sol in the cuvette & 0.67 & 4.5 & 81.0 & 0.33 & 2.13 & 19.0 \\
Gel in the cuvette & 0.86 & 3.85 & 85.0 & 0.14 & 1.5 & 5.5 \\
$\begin{array}{l}\text { Thin gel layer on the plate prior } \\
\text { to soaking }\end{array}$ & 0.99 & 4.5 & 95.5 & 0.01 & 14.2 & 4.5 \\
\hline
\end{tabular}

adsorption of $\mathrm{H}_{2} \mathrm{O}$ molecules, the adsorption of the probe molecules on the silane surface containing isolated $\mathrm{Si}-\mathrm{OH}$ groups, took place with prevalence of the AP neutral form, while on the surface containing geminal OH-Si-OH groups the protonated form prevailed [9].

The fluorescence emission spectra of aminopyrene at the concentration $2.1 \cdot 10^{-5} \mathrm{M}$ in water, ethanol, silane gel II and gel III were recorded. Lesser polarity of ethanol in relation to water induces a shift of the maximum emission of aminopyrene towards shorter waves from $\lambda_{\mathrm{em}}=440 \mathrm{~nm}$ to $\lambda_{\mathrm{em}}=425 \mathrm{~nm}$. Gel soaking in ethanol caused a further shifting of the maximum fluorescence emission to a shorter wavelength $\lambda_{\mathrm{em}}=415 \mathrm{~nm}$. No such phenomenon was observed for the plate stored in water. On this basis a conclusion may be drawn that a significant shift of the order of several nanometers towards shorter waves is induced by a total effect of the gel net and ethanol molecules on the fluorophore molecule. The ethanol molecules penetrate gel pores easier than water molecules, because they are less polar [9]. On the basis of the observed growth, and next decrease in the intensity of AP fluorescence emission after subsequent days of gel soaking both in ethanol and in water, it was found that part of the probe had not been covalently bonded, but adsorbed on the surface of gel pores being formed and eluted in time.

The fluorescence decay kinetics of aminopyrene in the sol and silane gel, obtained in a block and placed on the plate, was recorded. Decay curves were approximated by the diexpotential function:

$$
I(t)=A_{1} \exp \left(-t / \tau_{1}\right)+A_{2} \exp \left(-t / \tau_{2}\right) .
$$

Experimental curves recorded for the sol and gel are given in Table 2. In the silane sol the main aminopyrene domain of lifetime $4.5 \mathrm{~ns}$ was distinguished. In the gel this value was shortened to $3.85 \mathrm{~ns}$. This can be related to a decrease of polarity of the microenvironment of the probe molecule in the form of a gel net [12] and relaxation due to interactions with this net. This time is close to $3.93 \mathrm{~ns}$, which describes the lifetime of 


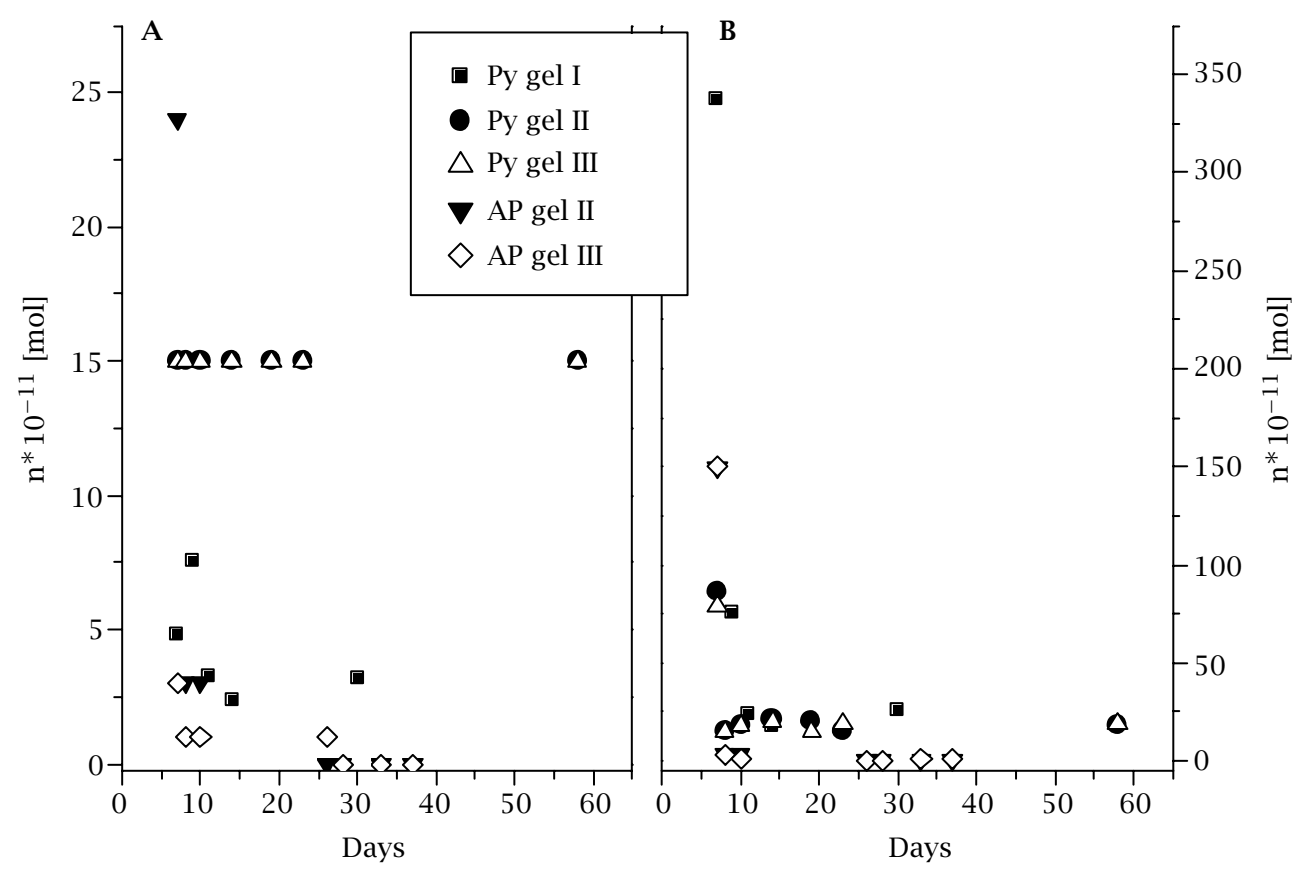

Figure 2. Elution of pyrene and aminopyrene by (A) water and by (B) ethanol from the gel with different compositions after subsequent days of soaking; $\mathrm{n}$-the amount of moles of the probe eluted from the gel.

Table 3. Properties of the silane carrier III on the plate with aminopyrene stored in the buffer at different $\mathrm{pH}$.

\begin{tabular}{|c|c|c|c|c|c|c|}
\hline \multicolumn{7}{|c|}{ Storage time [days] } \\
\hline & & 1 & & & 30 & \\
\hline $\mathrm{pH}$ & $\begin{array}{c}\text { Adherence } \\
{[\%]}\end{array}$ & $\begin{array}{c}\text { Transparency } \\
{[\%]}\end{array}$ & $\begin{array}{c}\text { Intensity of } \\
\text { fluorescence [a.u.] } \\
\lambda_{\mathrm{em}}=430 \mathrm{~nm}\end{array}$ & $\begin{array}{c}\text { Adherence } \\
{[\%]}\end{array}$ & $\begin{array}{c}\text { Transparency } \\
{[\%]}\end{array}$ & $\begin{array}{c}\text { Intensity of } \\
\text { fluorescence [a.u.] } \\
\lambda_{\mathrm{em}}=430 \mathrm{~nm}\end{array}$ \\
\hline 3.0 & 100 & 100 & 13.5 & 35 & 35 & 1.5 \\
\hline 4.5 & 100 & 100 & 15.0 & 35 & 35 & 8.0 \\
\hline 6.5 & 100 & 100 & 13.5 & 70 & 70 & 1.5 \\
\hline 9.5 & 100 & 100 & 17.0 & 70 & 70 & 22.0 \\
\hline 11.0 & 100 & 100 & 22.0 & 70 & 70 & 26.5 \\
\hline
\end{tabular}

$100 \%$ transparent film, smooth, without cracks, very good adhesion to the surface of glass.

$70 \%$ transparent film, smooth, with fine cracks on the film edges, good adhesion to the surface of glass.

$35 \%$ transparent film, smooth, with cracks, weak adhesion to the surface of glass.

aminopyrene in acetonitrile [13]. The values of $\tau_{1}$ and $\boldsymbol{\tau}_{2}$ (Table 2) for aminopyrene in silane gel placed on the plate, assume similar values to those obtained for AP connected through a hydrogen bond with silane surface $\tau_{1}=4.5 \mathrm{~ns}(95.5 \%)$ and $\tau_{2}=14.2(4.5 \%)$ [14]. This confirms the results of earlier studies that part of AP had been adsorbed on the surface of gel pores. The results are in agreement with the values of aminopyrene lifetime, which were obtained for this probe adsorbed in a commercially available gel that contained vicinal groups [14].

Figures $2 \mathrm{~A}$ and $2 \mathrm{~B}$ show results of the studies on pyrene and aminopyrene elution by ethanol and water from gels I, II, and III during 2 months. It was observed that the first two portions of ethanol eluted the biggest quantity of pyrene and unbounded aminopyrene (Figure 2B). The best results were obtained for gels II and III containing aminopyrene. The probe remained in the gel even after a month of storage in ethanol and water. Pyrene was eluted from the tested gels by water and ethanol gradually, in comparable amounts during the whole experiment.

Basing on the experimental results, for further testing gel III containing aminopyrene was selected. Taking into account a potential application of the tested silane carrier in the optical sensor for quick determinations in water solutions, a series of AP fluorescence measurements in the gel on the plate soaked in water at different pH during a month were taken (Table 3). It was found that after soaking the plates with the gel in the first 
portion of a buffer at different $\mathrm{pH}$, there was a shift of the maximum fluorophore emission of aminopyrene from $\lambda_{\mathrm{em}}=429 \mathrm{~nm}$ for the buffer at $\mathrm{pH} 3.0$ to $\lambda_{\text {em }}=426 \mathrm{~nm}$ for aminopyrene in the gel soaked in the buffers of $\mathrm{pH}$ above 9.00 . The highest intensity of fluorescence emission of the probe in the gel was observed for the plate soaked in the solution of $\mathrm{pH}$ 11.0. It did not change greatly after 30 days of soaking the plate with the gel in the buffer. Most of aminopyrene was eluted by the buffer at pH 3.0. Drastic storage conditions of the gels, i.e., strongly acidic environment, caused that the carrier layers on the plate after 30 days in the buffer of acidic pH partly cracked, which might induce the observed effect.

\section{CONCLUSIONS}

The experiments concerned elution of fluorescence probes, i.e., pyrene and aminopyrene with a silane gel of different compositions of the input sol, using water and ethanol. In the study a test consisting in storing the plates with deposited gel in a solvent for several hundred hours, was carried out. Best results were obtained for gel III containing aminopyrene. After 2-month storage in water or ethanol, this gel remained transparent and adhered to the plate surface. The emission intensity of the probe fluorescence in the gel enabled further determinations. Lower elution of aminopyrene was observed for the gel soaked in the solution at basic $\mathrm{pH}$.

The studies resulted in the selection of a silane carrier with proper composition to carry out further experiments with the construction of an optical sensor.

\section{ACKNOWLEDGMENTS}

The authors are grateful to the Committee for Scientific Reseaerch (Poland) for a financial support through grant no. 4 T08E 00924.

\section{REFERENCES}

[1] D. A. Ńives, Y. Zhang, and S. M. Angel, Anal. Chem. Acta 376 (1998), 235.

[2] C. D. Geddes, J. M. Chevers, and D. J. S. Birch, J. Fluoresc. 91 (1999), 73.

[3] K. K. Flora, M. A. Dabrowski, S. P. Musson, and J. D. Brennan, Can. J. Chem. 77 (1999), 1617.

[4] T. Yamanaka, Y. Takahashi, T. Kitamura, and K. Uchida, J. Luminescence 48-49 (1991), 265.

[5] Y. Xi, Z. Liangying, and W. Sasa, Sensor Actuators B 24-25 (1995), 347.

[6] S. Akimoto, A. Ohmori, and I. Yamazaki, J. Phys. Chem. B. 101 (1997), 3753.

[7] L. M. Ilharco, A. M. Santos, M. J. Silva, and J. M. G. Martinho, Langmuir. 11 (1995), 2419.

[8] I. Yamazaki, N. Tamai, and T. Yamazaki, J. Phys. Chem. 91 (1987), 3572.

[9] P. Hite, R. Krasnansky, and J. K. Thomas, J. Phys. Chem. 90 (1986), 5795.

[10] S. Pankasem and J. K. Thomas, J. Phys. Chem. 95 (1991), 7385.

[11] B. H. Milosavljevic and J. K. Thomas, J. Phys. Chem. 92 (1988), 2997.

[12] D. Avnir, D. Levy, and R. Reisfeld, J. Phys. Chem. 88 (1984), 5956.

[13] S. K. Saha and S. K. Dogra, J. Luminescence 75 (1997), 117.

[14] J. K. Thomas and E. H. Ellison, Adv. Coll. Inter. Scien. 89-90 (2001), 195. 


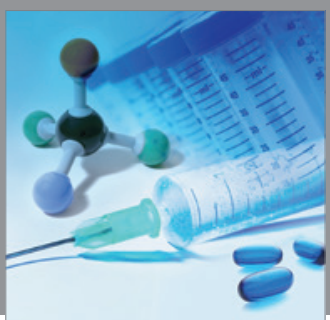

International Journal of

Medicinal Chemistry

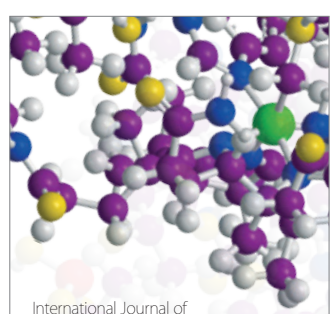

Carbohydrate Chemistry

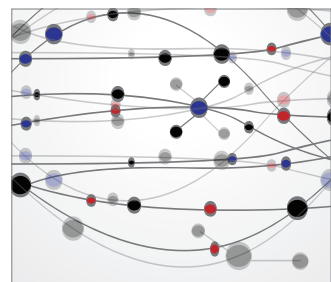

The Scientific World Journal
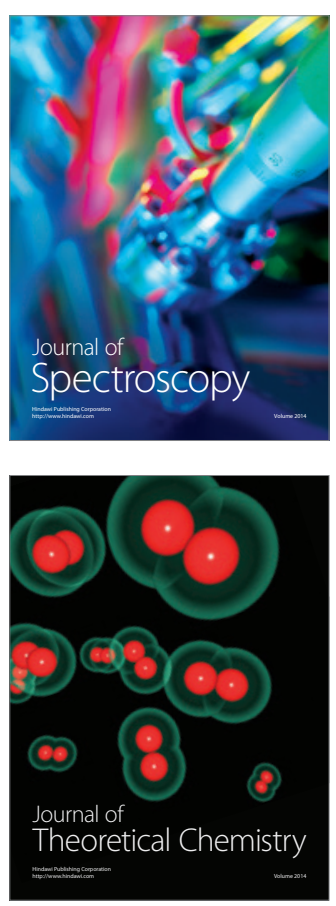
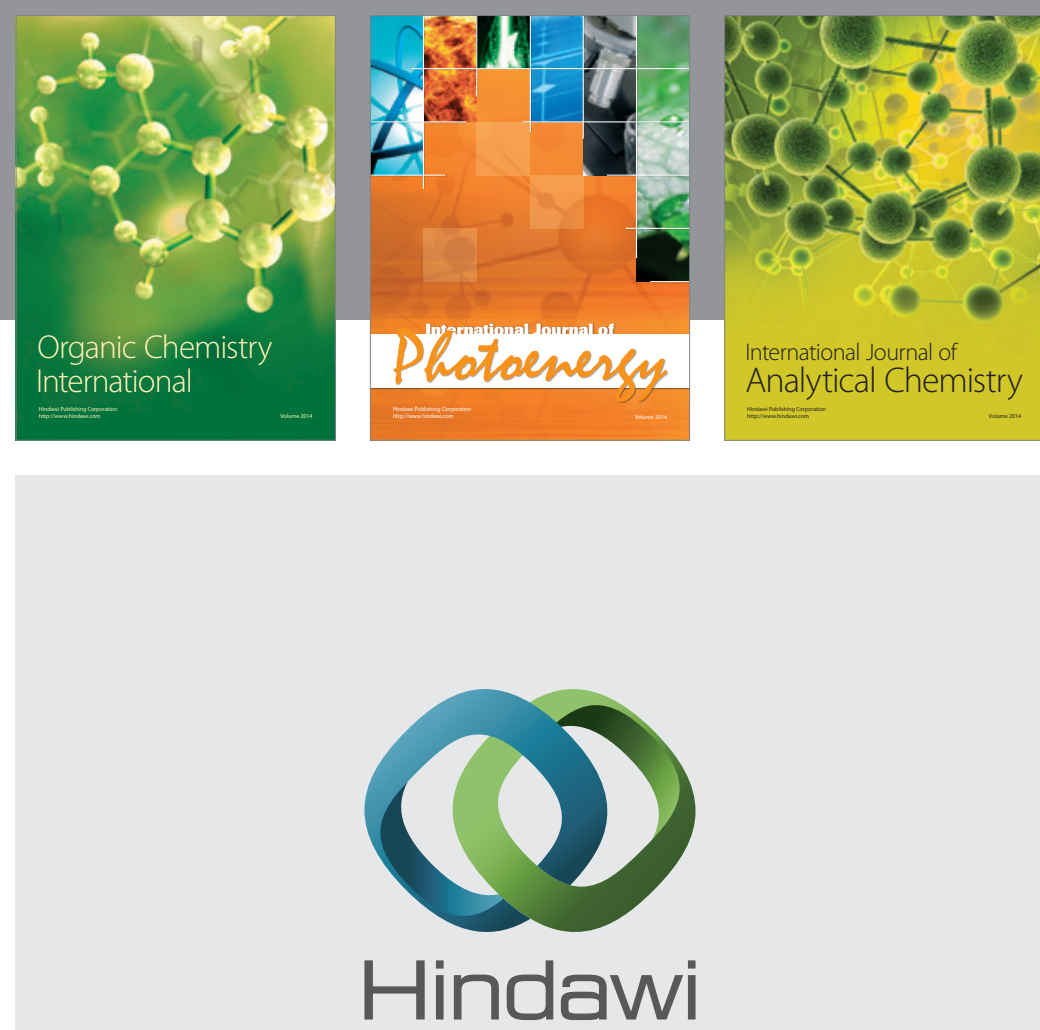

Submit your manuscripts at

http://www.hindawi.com
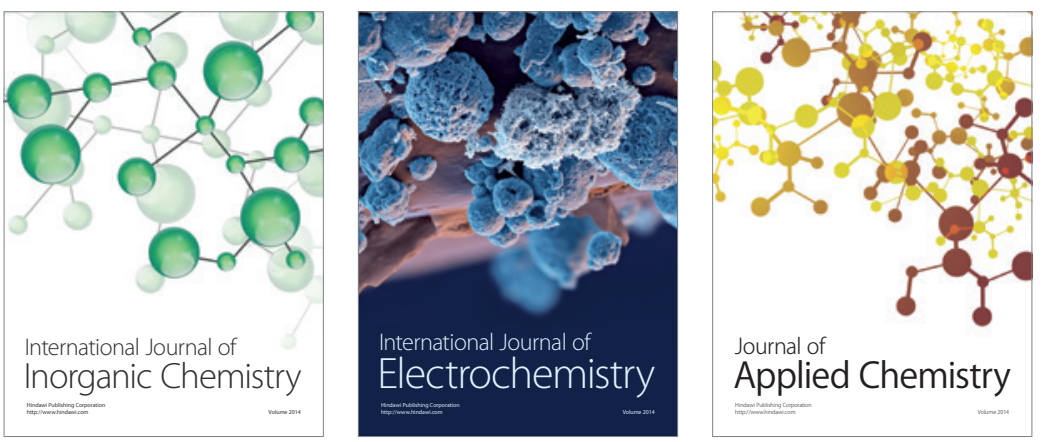

Journal of

Applied Chemistry
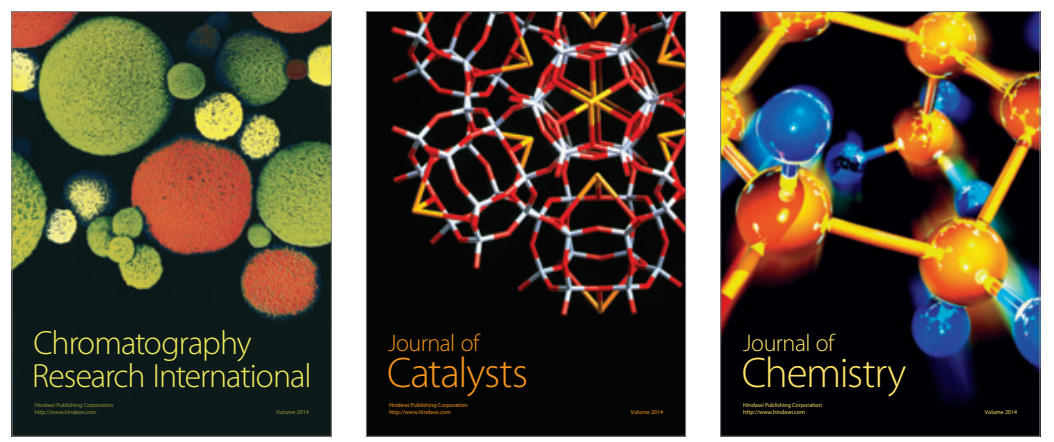
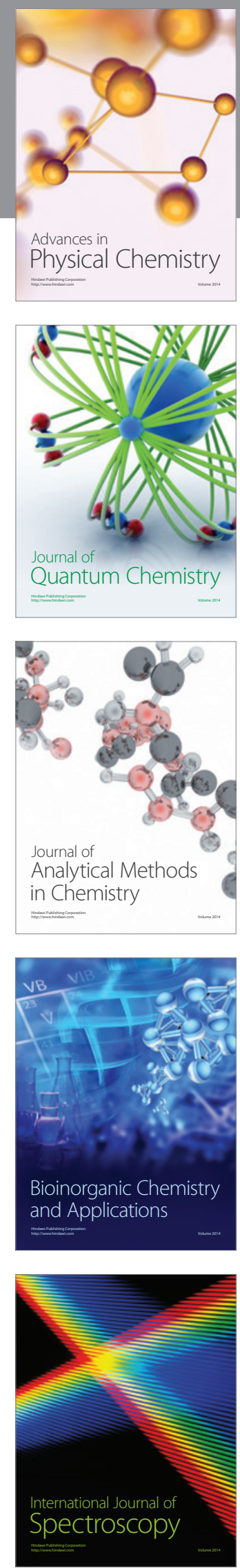